Asian J. Med. Biol. Res. 2020, 6 (2), 294-298; doi: 10.3329/ajmbr.v6i2.48074

\author{
Asian Journal of \\ Medical and Biological Research \\ ISSN 2411-4472 (Print) 2412-5571 (Online) \\ www.ebupress.com/journal/ajmbr
}

\title{
Article \\ Clinical investigation of different types of cases of outdoor patients using ultrasonography in Rangpur Medical College Hospital, Bangladesh
}

\author{
Shafaat Mahjabun ${ }^{1}$, Humayun Kabir ${ }^{2 *}$, Meftah Islam ${ }^{3}$, Abdur Rahim Azad ${ }^{4}$, Sumon Kumar Sarker ${ }^{1}$, Reza Tuhin \\ Hasinuzzaman $^{1}$, Syeda Olima Sultana ${ }^{1}$ and Mst. Nazmun Nahar ${ }^{1}$ \\ ${ }^{1}$ Department of Radiology and Imaging, Rangpur Medical College Hospital, Bangladesh \\ ${ }^{2}$ Deparnment of Veterinary Medicine, Tokyo University of Agriculture and Technology, Japan \\ ${ }^{3}$ Chittagong University of Engineering and Technology, Bangladesh \\ ${ }^{4}$ Chittagong Veterinary and Animal Sciences University, Bangladesh \\ *Corresponding author: Humayun Kabir, Department of Veterinary Medicine, Tokyo University of Agriculture \\ and Technology, Japan. E-mail: hkabirvet2@gmail.com
}

Received: 30 May 2020/Accepted: 27 June 2020/ Published: 30 June 2020

\begin{abstract}
Ultrasonography is an easy, noninvasive, rapid investigation advised by doctors worldwide. Hence we diagnosed different types of cases through ultrasonography at outdoor patients admitted to Department of Rangpur Medical College Hospital, Rangpur Bangladesh over a period of four months. Our objective was to investigate the prevalence of different types of cases diagnosed and evaluated by ultrasonography in a variety of physical conditions with multiple clinical scenarios. Ultrasonography were performed using GE Logiq V5 Expert ultrasound machine with linear and curved linear phased array transducers. A total 300 cases were diagnosed, where female and male cases were 204 (68\%), 96 (32\%) respectively. Most ultrasound examinations were abdominal, with the remainder being obstetric, pelvic, breast, and genitourinary examinations. In this study, abdominal ultrasonography represented 118 case (39.33\%), Pelvic 54 (18\%), KUB 57 (19\%), Breast 7 $(2.33 \%)$ and Obstetrics $64(21.33 \%)$. We found general clinical prevalence of abdominal cases were Cholelithiasis 17 (5.67\%), Cholecystitis 3 (1\%), Fatty liver 24 (8\%), Hepatic Mass 2 (0.67\%), Hepatic cyst 1 $(0.33 \%)$, Acute hepatitis $2(0.67 \%)$, Acute appendicitis $6(2 \%)$, Ascites $2(0.67 \%)$, Hernia 3(1\%), Acute pancreatitis $2(0.67 \%)$, Choledocholithiasis $2(0.67 \%)$, Splenomegaly $1(0.33 \%)$, Abscess $1(0.33 \%)$ and normal cases were 54 (18\%). In genitourinary, Stone 20 (6.67\%), Cyst 7 (2.33\%), RPD 11 (3.67), Cystitis 4 (1.33), Ectopic kidney $1(0.33 \%)$, Enlarged prostate $6(2 \%)$, Hydronephrosis $1(0.33 \%)$, Mass lesion $2(0.67 \%)$ and normal cases were 7 (2.33\%). In Pelvic, RPC 11 (3.67\%), Ovarian and adnexal cyst 11 (3.67\%), Uterine Anomaly $14(4.67 \%)$, Mass lesion $2(0.67 \%)$, Collection $7(2.33)$ and normal cases were $5(1.67 \%)$. In Obstetrics, normal pregnancy with no or minor complications, pregnancy with severe complications and abnormal pregnancy were 31(10.33\%), 30(10\%) and 3(1.0\%) respectively. In Breast, dilated duct $1(0.33 \%)$, Abscess $2(0.67 \%)$, Fibroadenoma $2(0.67 \%)$ and normal cases were $2(0.67 \%)$. Here, we found that Fatty liver, stone in genitourinary tract and gall stone were highly prevalent. So, health care providers need to concern and raise awareness to reduce the number of cases.
\end{abstract}

Keywords: ultrasonography; diagnosis; clinical; prevalence; retrospective study

\section{Introduction}

Ultrasonography is one of the new tools that we have acquired for diagnostic purpose. It is the brainchild of Dr. Ian Donald who was a gynecologist by profession. His invention has brought newer dimensions to the diagnostic field of not only obstetrics and gynecology but also to the other fields of medicine like internal medicine, surgery, ophthalmology, orthopedics, cardiology etc. and more and more fields are coming into its folds for not only diagnosing diseases but also for therapeutic purpose. It is a very popular modality with both 
the physicians as well as the patients. Reason being it is easily available, non-invasive, and with no radiation hazard. Also because some of the images that it displays on the screen are quite easily understood by the patients even. So that when a pregnant lady views her baby still inside her womb for the first time her joy and her husband's too is quite obvious. The thrill of seeing their baby moving, its heart beating, gives them so much of happiness. The basic principle of all ultrasound imaging is that, the ultrasound waves are send into the body, it is then reflected back from the interfaces of the organs and tissues. The reflected waves are then detected and displayed as an image on the monitor (Rashid, 2017).

Ultrasonography has been recommended for developing countries by the World Health Organization (WHO, 1985) since it is a technique that provides images immediately, is relatively inexpensive, can be carried out on an out-patient basis, and has no side effects. More than half of the world's population does not have access to at least some form of radiologic examination (Palmer, 1985) in developing countries, ultrasonography has become a classic tool.

Much has been written about the interest stimulated by ultrasonography in developing countries, (Clinical Ultrasound, 1990; Shaves, 1983; MacCready et al., 1982) on the economic implications of visual diagnosis in the Third World, (Kalifa et al., 1992; Fuch, 1991) or on specific pathologies, often tropical, in which ultrasonography has proven its worth (Houston, 1991). However, few studies have sought to determine the overall benefit that ultrasonography could provide as an aid to diagnosis and treatment (Raptopoulos et al., 1987; Mets, 1991; Doehring-Schwerdtfeger et al., 1992).

It was a retrospective study to investigate the clinical prevalence of different types of cases diagnosed and evaluated by ultrasonography.

\section{Materials and Methods}

The study was carried out at Radiology and Imaging Department of Rangpur Medical College Hospital, Rangpur, Bangladesh. The investigation was done during March to June 2019 as a part of fulfillment of one year long honorary training course in the mentioned department.

In this study we used GE logiq V5 expert ultrasonography machine having four probes, among which linear and curved linear probes were mostly used.

Among many patients who visited the outdoor department of Rangpur Medical College Hospital, some were advised for ultrasonography. The sonological investigation was requested by means of a special form which was completed by the person requesting ultrasonography (doctor). It included the identity of the patient, clinical data, the results of other investigations, and the organ to be examined. The presumed or suspected diagnosis, or the primary symptoms was also included. The test was done with paying of charge. The study comprises of male and female patients from all age group. Most patients were clinically stable, and some came with severe illness. A full bladder and overnight fasting were required to obtain good images specially for abdominal, pelvic, KUB and early pregnancy. In term pregnancy full bladder was only required when there was history of bleeding and placenta previa was suspected. Before performing the test, short history was taken, proper positioning was done, and the patients were properly exposed as per examination area.

Ultrasound is valuable in all trimesters of pregnancy. In the first trimester of pregnancy, ectopic pregnancy is a leading cause of mortality in women, requiring early identification and prompt intervention. Since clinical signs and symptoms are not reliable, ultrasound can play a pivotal role in its diagnosis. Early in their pregnancy, many women seek care for abdominal pain and/or vaginal bleeding.

\section{Results and Discussion}

In this clinical study, we found total 100 normal cases, which was $33.33 \%$, and abnormal cases were 200, which was about $66.67 \%$. And the normal and abnormal ratio were 1:2. In the investigation, female and male patient ratio were $204(68 \%)$ and $96(32 \%)$ respectively.

Among 300 cases, abdominal cases were 39.33\%, pelvic 18\%, KUB 19\%, obstetric $21.34 \%$ and breast $2.33 \%$ (Table 1). 
Table 1. Area wise percentage of the cases diagnosed by ultrasound ( 300 cases) are as follows:

\begin{tabular}{|l|l|l|l|l|l|}
\hline SL No & Distribution & Normal & Abnormal & Number of cases & Percentage (\%) \\
\hline 1 & Abdominal & 53 & 65 & 118 & 39.33 \\
\hline 2 & Pelvic & 7 & 47 & 54 & 18.00 \\
\hline 3 & KUB & 7 & 50 & 57 & 19.00 \\
\hline 4 & Breast & 2 & 5 & 7 & 2.33 \\
\hline 5 & Obstetrics & 31 & 33 & 64 & 21.34 \\
\hline \multicolumn{2}{|l}{ Total } & $\mathbf{1 0 0}$ & $\mathbf{2 0 0}$ & $\mathbf{3 0 0}$ & $\mathbf{1 0 0}$ \\
\hline
\end{tabular}

In abdominal scans, normal cases were 54 (18\%), abnormal cases were 65 (21.67\%), Most of the abnormal cases were fatty liver of different grades, cholelithiasis, acute appendicitis. Other cases comprise of cholecystitis, ascites, hernia, hepatic cyst, mass lesion, acute pancreatitis, splenomegaly and abscess (Table 2).

In pelvic scans 7 were normal with 47 abnormal cases. Most abnormal scan revealed uterine pathology which includes uterine fibroid, adenomyosis, bulky uterus, broadened cervix. Retained product of conception were found in 11 scans, and cyst in the ovary and adnexa were also 11. Mass lesion which were ovarian in origin were 2 in number. Collection in the cul-de-sac found in 7 pelvic scans which is an indicating marker for pelvic inflammatory disease in female.

In total 57 KUB scans 7 revealed no abnormality whereas 50 were showed abnormal findings. Among the scans most of the cases were stone in kidneys and ureters with or without complications like hydronephrosis, hydroureteronephrosis. Benign cystic lesions and benign enlargement of prostate gland in elderly patients were found in 7 and 6 cases respectively. Acute and chronic renal parenchymal disease revealed in 11 scans. Cystitis mostly found in the middle-aged man in about 4 cases and mass lesions were found in two cases one within the kidney another in the urinary bladder.

Table 2. General clinical prevalence of the cases diagnosed by ultrasound as follows:

\begin{tabular}{|c|c|c|c|c|}
\hline $\begin{array}{l}\text { SL } \\
\text { No. }\end{array}$ & Name of the cases & Distribution & $\begin{array}{l}\text { Number of } \\
\text { cases }\end{array}$ & $\begin{array}{l}\text { Percentage } \\
(\%)\end{array}$ \\
\hline 1 & Normal cases & \multirow{14}{*}{ Abdominal } & 54 & 18.0 \\
\hline 2 & Cholelithiasis & & 17 & 5.67 \\
\hline 3 & Cholecystitis & & 3 & 1.0 \\
\hline 4 & Fatty liver & & 24 & 8.0 \\
\hline 5 & Hepatic Mass & & 2 & 0.67 \\
\hline 6 & Hepatic cyst & & 1 & 0.33 \\
\hline 7 & Acute appendicitis & & 6 & 2.0 \\
\hline 8 & Ascites & & 2 & 0.67 \\
\hline 9 & Hernia & & 3 & 1.0 \\
\hline 10 & Acute pancreatitis & & 2 & 0.67 \\
\hline 11 & Choledocholithiasis & & 2 & 0.67 \\
\hline 12 & Acute hepatitis & & 2 & 0.67 \\
\hline 13 & Splenomegaly & & 1 & 0.33 \\
\hline 14 & Abscess & & 1 & 0.33 \\
\hline 15 & Normal cases & \multirow{9}{*}{ Genitourinary } & 7 & 2.33 \\
\hline 16 & Stone & & 20 & 6.67 \\
\hline 17 & Cyst & & 7 & 2.33 \\
\hline 18 & Renal parenchymal disease & & 11 & 3.67 \\
\hline 19 & Cystitis & & 4 & 1.33 \\
\hline 20 & Ectopic kidney & & 1 & 0.33 \\
\hline 21 & Enlarged prostate & & 6 & 2.0 \\
\hline 22 & Hydronephrosis & & 1 & 0.33 \\
\hline 23 & Mass lesion & & 2 & 0.67 \\
\hline 24 & Normal & \multirow{5}{*}{ Pelvic } & 5 & 1.67 \\
\hline 25 & Retained product of conception & & 11 & 3.67 \\
\hline 26 & Ovarian and adnexal Cyst & & 11 & 3.67 \\
\hline 27 & Uterine Anomaly & & 14 & 4.67 \\
\hline 28 & Mass lesion & & 2 & 0.67 \\
\hline
\end{tabular}




\begin{tabular}{|l|l|l|l|l|}
\hline 29 & Collection & & 7 & 2.33 \\
\hline 30 & Normal pregnancy with no complication & \multirow{3}{*}{ Obstetrics } & 31 & 10.33 \\
\cline { 5 - 6 } 31 & Pregnancy with moderate and severe complication & 30 & 10 \\
\cline { 4 - 5 } 32 & Abnormal pregnancy & Breast & 3 & 1.0 \\
\hline 33 & Normal & & 2 & 0.67 \\
\hline 34 & Abscess & & 2 & 0.67 \\
\hline 35 & Fibroadenoma & 2 & 0.67 \\
\hline 36 & Dilated duct & 1 & 0.33 \\
\hline \multicolumn{2}{|c|}{ Total } & $\mathbf{3 0 0}$ & $\mathbf{1 0 0}$ \\
\hline
\end{tabular}

In total, 64 obstetrics scans 31 revealed normal pregnancy without complications. Another 30 scans showed pregnancy with moderate to severe complications such as oligohydramnios in 16 scans, thick amniotic fluid in 7 scans, placenta previa 5 scans, and fetal death in 2 scans. Another 3 scans and showed abnormal pregnancy, 2 cases of ectopic pregnancy and a case of hydatidiform mole.

In breast scan, 2 scans were normal, 2 scans revealed breast abscess, 2 scans showing fibroadenoma which is common benign mass lesion and a case of dilated ducts more than $1.5 \mathrm{~mm}$ in diameter.

These results demonstrate that ultrasonography does represent an important contribution in determining a diagnosis and the choice of treatment. In this study, we found that, high prevalent cases (8\%) were NAFLD (fatty liver of different grades), which had the similarities with the findings of (Kirovski et al., 2010) who showed the prevalence of ultrasound-diagnosed NAFLD was $40.0 \%$. Genitourinary tract stone prevalence in the study was $6.67 \%$ that correlates with the findings by One report performed by (Bansal et al., 2009) who revealed the prevalence of all kidney stones in their study was $8.6 \%$. In our study, we found the prevalence of gallstones $5.67 \%$ which correlates with the findings of (Alshoabi, 2016) who reported the prevalence of gallstones in his study was $18-23.5 \%$. We found that, prevalence rate of ovarian and adnexal cysts were comprise of 3.67\%, which had the similarities with the findings of (Sadowski et al., 2018) who showed the prevalence of ultrasound-diagnosed of indeterminate adnexal cysts in their study was $23.9 \%$.

\section{Conclusions}

In our clinical investigation, the major cases were fatty liver, gall stone and renal stone. The results are very promising which could aid in the detection of the early stage of the diseases, such as in asymptomatic patients with fatty liver, the early diagnosis can prevent the progression to major lesions such as steato-hepatitis or even cirrhosis, early detection of gall stone can prevent cholecystitis, mucocele of gall bladder or even mass lesion, early identification of renal stone can prevent obstructive nephropathy. Ultrasound is now also used for therapeutic purpose as for crushing gall bladder and renal stones. And about obstetric scans we can say, though the government is taking many steps for pregnant mothers but the prevalence of pregnancy related complications are not less. So, there is need to raise public awareness by physicians.

\section{Acknowledgements}

We would like to express our gratitude to Department of Radiology and Imaging, Rangpur Medical College Hospital (RpMCH) for supporting this research work.

\section{Conflicts of interest}

None to declare.

\section{Reference}

Bansal AD, J Hui and DS Goldfarb, 2009. Asymptomatic nephrolithiasis detected by ultrasound. Clin. J. Am. Soc. Nephrol., 4: 680-684.

Chaves OE, 1983. How to introduce diagnostic ultrasound in medical institutions of limited resources. Ultrasound Med. Biol., 1: 95-97.

Clinical ultrasound in developing countries (editorial), 1990. Lancet, 336: 1225-1226.

Doehring-Schwerdtfeger E, IM Abdel-Rahim, M Dittrich, Q Mohamed-Ali, D Franke, R Kardorff, J Richter and JHH Ehrich, 1992. Ultrasonography as a diagnostic aid for a district hospital in the tropics. Am. J. Trop. Med. Hyg., 46: 727-731.

EA Sadowski, V Paroder, K Patel-Lippmann, JB Robbins, L Barroilhet, E Maddox, T McMahon, E Sampene, AP Wasnik, AD Blaty and E Katherine, 2018. Maturen, indeterminate adnexal cysts at US: prevalence and characteristics of ovarian cancer. Radiology, 287: 1041-1049. 
Fuchs WA, 1991. Radiology in developing countries. Invest Radiol., 26: 906-909.

Kirovski G, D Schacherer, H Wobser, H Huber, C Niessen, C Beer, J Schölmerich and C Hellerbrand, 2010. Prevalence of ultrasound-diagnosed non-alcoholic fatty liver disease in a hospital cohort and its association with anthropometric, biochemical and sonographic characteristics. Int. J. Clin. Exp. Med., 3: 202-210.

Houston S, 1991. Ultrasound: appropriate technology for tropical field work. Trans. R. Soc. Trop. Med. Hyg., 85: 321-323.

Kalifa G, A Bouras, A Reymond-Yeni and D Gendrel, 1992. Imagerie en pJdiatrie. StratJgie et implications Jconomiques pour le tiers-monde. Ann Pediatr., 139: 67-70.

McCready VR, DO Cosgrove and R Hill, 1982. Symposium on ultrasound in developing countries. Ultrasound, 82: 81-85.

Mets T, 1991. Clinical ultrasound in developing countries. Lancet, 337: 337-358.

Palmer PES, 1985. The epidemic of investigations. Int. J. Epidemiol., 14: 359-362.

Raptopoulos V, RJ Goldberg, EH Smith and RC Effison, 1987. Clinician's appraisal of sonography. Radiology, 165: 237-239.

Rashid SQ, 2017. The basics of ultrasonography. Bangladesh Medical Journal., Jan, 46 (1).

Report of a WHO Scientific Group, 1985. Future use of new imaging technologies in developing countries. World Health Organ Tech Rep Ser., 723.

Alshoabi S, 2016. Gallstones: Site, Size, Number, Prevalence and Complications by Ultrasonography. Int. J. Med. Imaging, 4: 52-56. 